\title{
When Feedback Backfires: Influences of Negative Discrepancies Between Physicians' Self and Assessors' Scores on Their Subsequent Multisource Feedback Ratings
}

Citation for published version (APA):

van der Meulen, M. W., Arah, O. A., Heeneman, S., Egbrink, M. G. A. O., van der Vleuten, C. P. M., \& Lombarts, K. M. J. M. H. (2021). When Feedback Backfires: Influences of Negative Discrepancies Between Physicians' Self and Assessors' Scores on Their Subsequent Multisource Feedback Ratings. Journal of Continuing Education in the Health Professions, 41(2), 94-103.

https://doi.org/10.1097/CEH.0000000000000347

Document status and date:

Published: 01/01/2021

DOI:

10.1097/CEH.0000000000000347

Document Version:

Publisher's PDF, also known as Version of record

Document license:

Taverne

Please check the document version of this publication:

- A submitted manuscript is the version of the article upon submission and before peer-review. There can be important differences between the submitted version and the official published version of record. People interested in the research are advised to contact the author for the final version of the publication, or visit the DOI to the publisher's website.

- The final author version and the galley proof are versions of the publication after peer review.

- The final published version features the final layout of the paper including the volume, issue and page numbers.

Link to publication

\footnotetext{
General rights rights.

- You may freely distribute the URL identifying the publication in the public portal. please follow below link for the End User Agreement:

www.umlib.nl/taverne-license

Take down policy

If you believe that this document breaches copyright please contact us at:

repository@maastrichtuniversity.nl

providing details and we will investigate your claim.
}

Copyright and moral rights for the publications made accessible in the public portal are retained by the authors and/or other copyright owners and it is a condition of accessing publications that users recognise and abide by the legal requirements associated with these

- Users may download and print one copy of any publication from the public portal for the purpose of private study or research.

- You may not further distribute the material or use it for any profit-making activity or commercial gain

If the publication is distributed under the terms of Article 25fa of the Dutch Copyright Act, indicated by the "Taverne" license above, 


\title{
When Feedback Backfires: Influences of Negative Discrepancies Between Physicians' Self and Assessors' Scores on Their Subsequent Multisource Feedback Ratings
}

Mirja W. van der Meulen, PhD; Onyebuchi A. Arah, MD, PhD; Sylvia Heeneman, PhD; Mirjam G. A. oude Egbrink, PhD; Cees P. M. van der Vleuten, PhD; Kiki M. J. M. H. Lombarts, PhD

Introduction: With multisource feedback (MSF) physicians might overrate their own performance compared with scores received from assessors. However, there is limited insight into how perceived divergent feedback affects physicians' subsequent performance scores.

Methods: During 2012 to 2018, 103 physicians were evaluated twice by 684 peers, 242 residents, 999 coworkers, and themselves in three MSF performance domains. Mixed-effect models quantified associations between the outcome variable "score changes" between first and second MSF evaluations, and the explanatory variable "negative discrepancy score" (number of items that physicians rated themselves higher compared with their assessors' scores) at the first MSF evaluation. Whether associations differed across assessor groups and across a physician's years of experience as a doctor was analyzed too.

Results: Forty-nine percent of physicians improved their total MSF score at the second evaluation, as assessed by others. Number of negative discrepancies was negatively associated with score changes in domains "organization and (self)management" ( $\mathrm{b}=-0.02 ; 95 \%$ confidence interval [Cl], -0.03 to $-0.02 ; \mathrm{SE}=0.004)$ and "patient-centeredness" $(\mathrm{b}=-0.03 ; 95 \% \mathrm{Cl},-0.03$ to -0.02 ; SE $=0.004$ ). For "professional attitude," only negative associations between score changes and negative

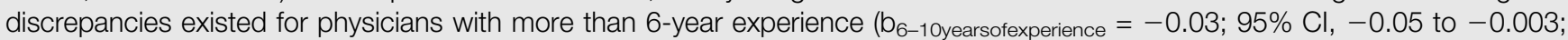
$\mathrm{SE}=0.01 ; b_{16-20 y e a r s o f e x p e r i e n c e}=-0.03 ; 95 \% \mathrm{Cl},-0.06$ to $-0.004 ; \mathrm{SE}=0.01$ ).

Discussion: The extent of performance improvement was less for physicians confronted with negative discrepancies. Performance scores actually declined when physicians overrated themselves on more than half of the feedback items. PA score changes of more experienced physicians confronted with negative discrepancies and were affected more adversely. These physicians might have discounted feedback due to having more confidence in own performance. Future work should investigate how MSF could improve physicians' performance taking into account physicians' confidence.

Keywords continuing professional development, multisource feedback, performance improvement, physicians' professional performance, score changes, experience, workplace-based assessment

DOI: $10.1097 / \mathrm{CEH} .0000000000000347$

\begin{abstract}
Disclosures: The authors declare no conflict of interest.
$M$.W. van der Meulen was the initiator of this study and responsible for the conception of this study, draft of the study design, and analysis and interpretation of the data for this work. O. A. Arah additionally contributed to the study design and analysis of the data. S. Heeneman, M. G. A. oude Egbrink, C. P. M. van der Vleuten, and K. M. J. M. H. Lombarts made contributions to the study design. M. W. van der Meulen wrote all the drafts of this work, which were revised with input from all authors. All authors contributed to critically revising the paper for important intellectual content. All authors gave their final approval for this research paper to be submitted on their behalf. All authors agree to be accountable for all aspects of the work in ensuring that questions related to the accuracy or integrity of any part of the work are appropriately investigated and resolved.
\end{abstract}

Supplemental digital content is available for this article. Direct URL citations appear in the printed text and are provided in the HTML and PDF versions of this article on the journal's Web site (www.jcehp.org).

A waiver of informed consent was provided by the institutional review board of the Academic Medical Center of the University of Amsterdam, the Netherlands, reference number W12_267.

Dr. van der Meulen: is PhD Candidate, Department of Educational Development and Research, Faculty of Health, Medicine and Life Sciences, School of Health Professions Education, Maastricht University, Maastricht, the Netherlands, and Professional Performance and Compassionate Care Research Group, Department of Medical Psychology, Amsterdam University Medical Centers, University of Amsterdam, Amsterdam, the Netherlands. Dr. Arah: is professor, Department of Epidemiology, University of California, Los Angeles (UCLA), Los Angeles, the United States of America. Dr. Heeneman: is professor, Department of Pathology, Faculty of Health, Medicine and Life Sciences, School of Health Professions Education, Cardiovascular Research Institute Maastricht, Maastricht University, Maastricht, the Netherlands. Dr. oude Egbrink: is professor, Department of Physiology, Faculty of Health, Medicine and Life Sciences, School of Health Professions Education, Maastricht University, Maastricht, the Netherlands. Dr. van der Vleuten: is professor, Department of Educational Development and Research, Faculty of Health, Medicine and Life Sciences, School of Health Professions Education, Maastricht University, Maastricht, the Netherlands. Dr. Lombarts: is professor, Professional Performance and Compassionate Care Research Group, Department of Medical Psychology, Amsterdam University Medical Centers, University of Amsterdam, Amsterdam, the Netherlands.

Correspondence: Mirja W. van der Meulen, Professional Performance and Compassionate Care Research Group, Amsterdam University Medical Centers, University of Amsterdam, Amsterdam, The Netherlands, Meibergdreef 9, PO Box 227001100 DD Amsterdam, The Netherlands; e-mail: m.w.vandermeulen@amsterdamumc.nl.

Copyright $\odot 2021$ The Alliance for Continuing Education in the Health Professions, the Association for Hospital Medical Education, and the Society for Academic Continuing Medical Education 
T he evaluation of physicians' competence and performance is a key issue in current research and policy agendas. ${ }^{1-4}$ This is not surprising, given that high-quality patient care needs high-performing physicians, which asks for regular evaluation. Workplace-based assessment methods enable the regular evaluation of physicians' professional performance in daily practice. $^{5}$ One popular method is the use of multisource feedback (MSF), where information about a physician's professional performance is collected using items rated by multiple assessors and assessor groups, ${ }^{6-8}$ such as peers, coworkers, patients, and the physicians themselves. These combined evaluations from multiple groups are essential as the goal is to assess physicians' integral professional performance, consisting of the complex and integrated interplay between the use of knowledge, skills, attitudes, and values. ${ }^{9-11}$ The collected feedback is believed to help physicians improve their professional performance because it can reveal shortcomings in current performance, while current performance can also be praised. ${ }^{12-14}$ Indeed, follow-up research on physicians who participated in MSF showed positive results: physicians reported to have changed their performance after receiving and reflecting upon feedback. ${ }^{15-24}$

However, the effect of MSF can be 2-fold: it can be constructive and destructive. ${ }^{25}$ The extent of MSF's effect on physicians' practice depends on numerous factors such as the characteristics of feedback, feedback recipients, and feedback providers. ${ }^{6} 14,18,21,25,26$ When receiving MSF, physicians can be confronted with feedback that is incongruent with their own performance beliefs. These discrepancies can either be positive when the self-assessment scores turn out to be lower than the scores received from assessors or negative, in which the selfassessment scores are higher than assessors' scores. Especially, negative discrepancies between self-assessment scores and assessors' assessment scores could affect physicians' subsequent performance. These discrepancies may stimulate physicians in undertaking actions for performance change because they reveal current, unknown, performance gaps. Alternatively, when confronted with too many negative discrepancies, physicians' acceptance of feedback might become challenging due to emotional responses. ${ }^{27-29}$

Furthermore, feedback recipients' confidence, experience, and how credible they perceive the feedback source also influence the acceptance of incongruent feedback. ${ }^{20,26-28,30,31}$ The right amount of confidence creates opportunities to accept potentially threatening appraisals, yet too much confidence creates tension in accepting feedback that is incongruent with one's perception. ${ }^{26}$ Feedback recipients' experience is directly linked to this confidence: the more experienced, the more confident. ${ }^{26}$ Furthermore, accepting incongruent feedback is also affected by the perceived credibility of the feedback source. If the assessor is not deemed credible, acceptance of feedback becomes challenging as well. ${ }^{26-28}$ This source's credibility is related to the type of assessor group, and the type of performance domain this assessor assesses. Physicians are more likely to accept feedback on clinical competence from assessors who are indeed capable of commenting on clinical competence. ${ }^{31}$

Multiple factors can influence the uptake and acceptance of MSF and exploring these influences can assist physicians in their continuous professional development. One of these influencing factors is the incongruence between self-assessment scores and those of other assessors. Until recently, little is known about the association of negative discrepancies with physicians' actual MSF score changes. Because MSF ratings provided by colleagues often poorly correlated with selfratings, it is specifically interesting to look at how negative discrepancies influence physicians' score changes in MSF. ${ }^{27}$ To get a grasp of this, possible association requires investigating the factors that might moderate or explain these associations, such as physicians' experience and the different assessor groups. Knowing how negative discrepancies influence physicians' subsequent MSF scores is crucial to tailoring the MSF in helping physicians accept incongruent feedback. ${ }^{32,33}$ Therefore, this study examined the associations of negative discrepancies of physicians' self-assessment scores and the scores they received from assessors with their score changes in subsequent MSF evaluations. The current study specifically addressed the following questions: (1) Are negative discrepancies between physicians' self-assessment scores and assessors' scores associated with score changes in a subsequent MSF evaluation? (2) Do physicians' years of experience and the feedback source modify these possible associations? This longitudinal observational study could give further insights into the potential contributions of MSF to the evaluation and improvement of physicians' professional performance.

\section{METHODS}

\section{Study Setting and Design}

This observational retrospective study was conducted in the Netherlands, where physicians participated in a performance appraisal process between 2012 and 2018 using MSF. Since 2008, physicians' participation rate in MSF evaluation, but not their scores or rankings, has been monitored and published to the public by the Dutch Inspectorate of Health. Dutch physicians are encouraged to participate in an MSF performance appraisal process and can select from various MSF instruments for this purpose. Physicians who chose to evaluate their performance using the validated MSF instrument "INviting Coworkers to Evaluate Physicians-Tool" (INCEPT) ${ }^{34}$ were made aware that their data could be used for research purposes. The INCEPT tool was developed as a cocreation of researchers and practicing physicians to support physicians' professional performance improvement, first, by providing the MSF scores to the evaluated physician and, second, by conducting research upon physicians' performance data resulting from this tool.

The Institutional Review Board of the Amsterdam University Medical Centers, location AMC, provided a waiver of informed consent for this study.

\section{Participants and Data Collection}

Between 2012 and 2018, 2413 Dutch physicians from multiple hospitals and departments participated in the MSF program with the validated INCEPT instrument. Data collection of evaluated physicians occurred online; data were exported anonymously to the primary researcher by a trusted third party. The trusted third party, Empirion/Medox.nl, is the web developer of the tool and is the only entity that collects and has access to the MSF data. Legal agreements per Dutch law on 
participants' privacy have been made with Empirion/Medox.nl about the anonymity and export of the data. For this study, 103 physicians were included, as these physicians participated twice (in the same role/discipline as medical specialist) in the MSF program between 2012 and 2018, assessed themselves at both time points, and had received sufficient evaluations from all respondent groups on both evaluations for reliable MSF scores. Previous research determined that a minimum of three residents, three peers, and four coworkers are needed for a reliable average score using a SEM of $0.26 .{ }^{34}$ SEM can be used to create a confidence interval (CI) around scores. ${ }^{35}$ An SEM value of 0.26 was set as the smallest allowable value for a $95 \% \mathrm{CI}$ interpretation $(1.96 \times 0.26 \times 2 \approx 1)$, representing a $95 \%$ CI of \pm 0.5 around the average score. ${ }^{36,37}$ At both time points, physicians collected their feedback data within one month, by selecting and inviting assessors to provide anonymous feedback on their performance using the INCEPT MSF tool in an SSLcertified web-based environment. Physicians were instructed to invite at least eight peers (medical colleagues), eight coworkers (other health care professionals, such as nurses and [paramedical] assistants), and eight residents to evaluate them. These assessors were contacted per email, stressing the formative purpose and the confidential, anonymous, voluntary character of the evaluation. The physicians were asked to self-evaluate their performance as well using the same questionnaire and to provide information about themselves, such as age, gender, experience (years certified as medical specialist), and years of employment as a medical specialist. When more than four assessors per assessor group provided feedback, physicians received a personalized feedback report at the end of the MSF evaluation period. This feedback report contained anonymized aggregated scores per assessor group, narrative comments per assessor group, and physician's self-assessment scores. Within this report, aggregated assessor and self-assessment scores were graphically depicted for each feedback item, showing the discrepancy between physicians' self-assessment and others' scores (see Figure 1, Supplemental Digital Content 1, http:// links.lww.com/JCEHP/A114). Physicians reviewed their report and identified areas for improvement accompanied by a formal follow-up with a facilitator outside of the organization or clinical department. The facilitators encouraged and supported physicians to use the feedback for developmental goals. The feedback report was sent to the physician only; neither head of departments nor external institutions such as the health inspectorate received the report. The reports were meant to be used as formative feedback and not have a role in any (highstakes) decisions.

\section{Measurements}

\section{Outcome Variables}

The primary outcome variable in this study was physicians' "score change" between the first MSF evaluation (time 1) and the second MSF evaluation (time 2). This continuous variable ranges from -4 to 4 , with negative values indicating that time 2 scores declined compared with time 1 scores and positive values indicating that time 2 scores were higher compared with time 1 scores. This score change was calculated for three different performance domains, resulting in three outcome variables. The MSF questionnaire INCEPT covers these three performance domains and contains 18 specific items and three global rating items about physicians' professional performance. The three performance domains are "professional attitude" (PA), "organization and (self)management" (OSM), and "patientcenteredness" (PC). Representative items of the PA, OSM, and PC domains are, for example: "Shows respect to other health care professionals," "Maintains quality medical records," and "Shows compassion to patients," respectively (see Supplemental Digital Content 1, http://links.lww.com/JCEHP/A114 for a complete overview of item-clustering). All 18 items and the global rating items were rated on a 5 -point Likert scale $(1=$ totally disagree, 2 = disagree, $3=$ neutral, 4 = agree, 5 = totally agree) with an additional "I cannot judge this statement" option. To obtain domain-specific score changes, we subtracted average PA, OSM, and PC scores obtained at time 1 from time 2 scores. The domain scores were calculated per assessor group and aggregated per assessor group to obtain the mean performance score in each domain. Results from our previous research indicated that the clustering of the items differed per assessor group. ${ }^{34}$ We thus calculated domain-specific average scores, according to the clustering of the specific type of assessor group. MSF scores were only aggregated for physicians who received sufficient evaluations to obtain reliable domainspecific scores for formative feedback use.

\section{Explanatory Variables}

Three explanatory variables were included in the model. These variables were the number of negative discrepancies, type of assessor group, and physicians' years of experience. First, we computed the total number of negative discrepancies between self-assessment scores and other-scores per physician, per assessor group, by determining how many of the 18 items a physician had overrated his or her performance. We calculated the discrepancy score by subtracting the physician's selfassessment scores with the assessors' scores (per assessor group) for each of the 18 items. When overrating occurred on an item (self-assessment scores being higher than assessors' scores), a score of one was given, and by summing these scores, a total negative discrepancy score was created. We chose to calculate this total negative discrepancy score to capture how often physicians were confronted with a negative discrepancy in their feedback report, regardless of how large this discrepancy was. This score ranged from zero to 18 , with zero indicating no negative discrepancy and 18 indicating that negative discrepancies occurred for all items. For example, if a physician overrated his/her performance on six items compared with the score received by residents, this physician received a six for the negative discrepancy score on the resident level. The second variable was the type of assessor group, which was operationalized as a nominal variable, with three different groups: peers, residents, and coworkers. Third, we operationalized physicians' experience (years certified as medical specialist) as an ordinal variable with five values ranging from 0 to 5 years, 6 to 10 years, 11 to 15 years, 16 to 20 years, and more than 21 years.

\section{Covariates}

A covariate that had to be adjusted for in the model is the score physicians received from assessors in the first MSF evaluation: the so-called prescore or "scores at time 1." Also, we included "the number of months between the first and second MSF evaluation." Additionally, we included "the percentage of missing values per performance domain" (the number of 
assessors who opted for "I cannot judge this statement" per item divided by the number of assessors for that physician, aggregated to the performance domain). These missing values on items were not imputed but were incorporated as covariates "the percentage of item missingness" for the MSF evaluation during time 1 and time 2. Furthermore, as physicians' gender was found to be associated with overrating their performance (male physicians tend to overrate more ${ }^{19}$ ), this variable was incorporated as a covariate as well.

\section{Data Analyses}

Descriptive statistics were calculated for the characteristics of the study population. Outliers were explored with Mahalanobis distance and removed if deemed suitable, normality and heterogeneity of the data were examined using standardized residuals. ${ }^{38}$ Evaluations with more than $50 \%$ missing values on the 18 items were removed and not included in data analyses. The remaining evaluations with missing values were aggregated to use for analyses, with the percentage of missing values included as covariates. To quantify possible associations between the negative discrepancy score and performance score changes between time 1 and time 2, we used three, linear, mixed-effects, regression models estimated with maximum likelihood method and Satterthwaite method for $t$ tests. The linear mixed models with random effects allowed for the adjustment of hierarchical clustering of multiple evaluations within physicians. First, we modeled how much variance was associated with the differences between physicians on the primary outcome variables to determine the intraclass correlation, a practical value to establish whether multilevel modeling is required or not. ${ }^{39}$ We investigated whether and how physicians' performance score changes as rated by their assessors would be associated with negative discrepancies by adding the negative discrepancy score variable to the model. Next, we added the type of assessor group in interaction terms to the model to investigate whether the type of assessor group modified the associations between negative discrepancies and performance score changes. Finally, we used random-slope mixed models to investigate variation in how physicians "deal" with negative discrepancies (namely, the associations of negative discrep- ancies with assessors' score changes). If the random-slope models showed better fit than the random-intercept model (indicating that the associations between negative discrepancies and score change differed by physician), a cross-level interaction effect with physicians' years of experience was added. This cross-level interaction was added to investigate whether physicians' experience could explain these random slopes. To interpret the regression coefficients and to avoid multicollinearity between explanatory variables, we centered the continuous explanatory variables to the grand means. ${ }^{38} \mathrm{We}$ applied a Bonferroni correction for multiple testing. We used $\mathrm{R}$ studio version 3.5.1 with packages "lme4," "lmerTest," "ggplot2," and "psych" for data analyses. ${ }^{40}$

\section{RESULTS}

\section{Study Participants}

One hundred and three physicians from 42 departments in nine hospitals participated twice in an MSF procedure, including self-assessments. For these physicians, 3182 evaluations were filled out by assessors (excluding 88 evaluations with more than 50\% missing items): 1522 at time 1 and 1660 at time 2. Physicians had an average total self-score of 4.16 $(S D=0.36)$ at time 1 , and an average total self-score of 4.18 $(S D=0.39)$ at time 2 . Per assessor type, physicians on average overrated themselves on $6.19(\mathrm{SD}=4.27), 6.55(\mathrm{SD}=4.47)$, and 6.15 (SD = 4.77) items compared with residents, peers, and coworkers. On average, each physician received 15 evaluations at time 1 and 16 evaluations at time 2 . The response rates per assessor group at time 1 and time 2 MSF were $86 \%$ and $81 \%$ for residents, $83 \%$ and $86 \%$ for peers, and $84 \%$ and $83 \%$ for coworkers, respectively. From the total evaluation data, $9.4 \%$ was missing. Sixty-six percent of the evaluated physicians were male, mostly from the age category of 36 to 40 years, with 1 to 10 years of experience on average. Forty-nine percent of the physicians had improved their total MSF score between time 1 and time 2, according to all assessor groups. Table 1 summarizes physicians' and assessors' characteristics, and Table 2 summarizes the average scores that physicians received from their assessors.

\section{TABLE 1.}

\section{Descriptive Statistics of the Evaluated Physicians and Their Assessors}

\begin{tabular}{|c|c|c|c|c|}
\hline & \multirow[b]{2}{*}{ Evaluated Physicians } & \multicolumn{3}{|c|}{ Assessors Who Evaluated } \\
\hline & & Residents & Peers & Coworkers \\
\hline$N(N$ evaluations) & 103 (3182) & 242 & 684 & 999 \\
\hline \multicolumn{5}{|c|}{$\%$ Participants of age category } \\
\hline$<25 y$ & $0 \%$ & $0.4 \%$ & $0.3 \%$ & $1.4 \%$ \\
\hline $25-35$ y & $9.7 \%$ & $72.7 \%$ & $4.4 \%$ & $18.7 \%$ \\
\hline $36-40 y$ & $21.4 \%$ & $12.8 \%$ & $21.3 \%$ & $11.3 \%$ \\
\hline $51-55$ y & $16.5 \%$ & $1.7 \%$ & $12.6 \%$ & $14.8 \%$ \\
\hline $56-60$ y & $15.5 \%$ & $0 \%$ & $9.1 \%$ & $10.8 \%$ \\
\hline $61-80 y$ & $2.9 \%$ & $0.4 \%$ & $5.1 \%$ & $4.8 \%$ \\
\hline missing & $1 \%$ & $9.1 \%$ & $13.3 \%$ & $8.9 \%$ \\
\hline
\end{tabular}




\section{TABLE 1.}

Descriptive Statistics of the Evaluated Physicians and Their Assessors (Continued)

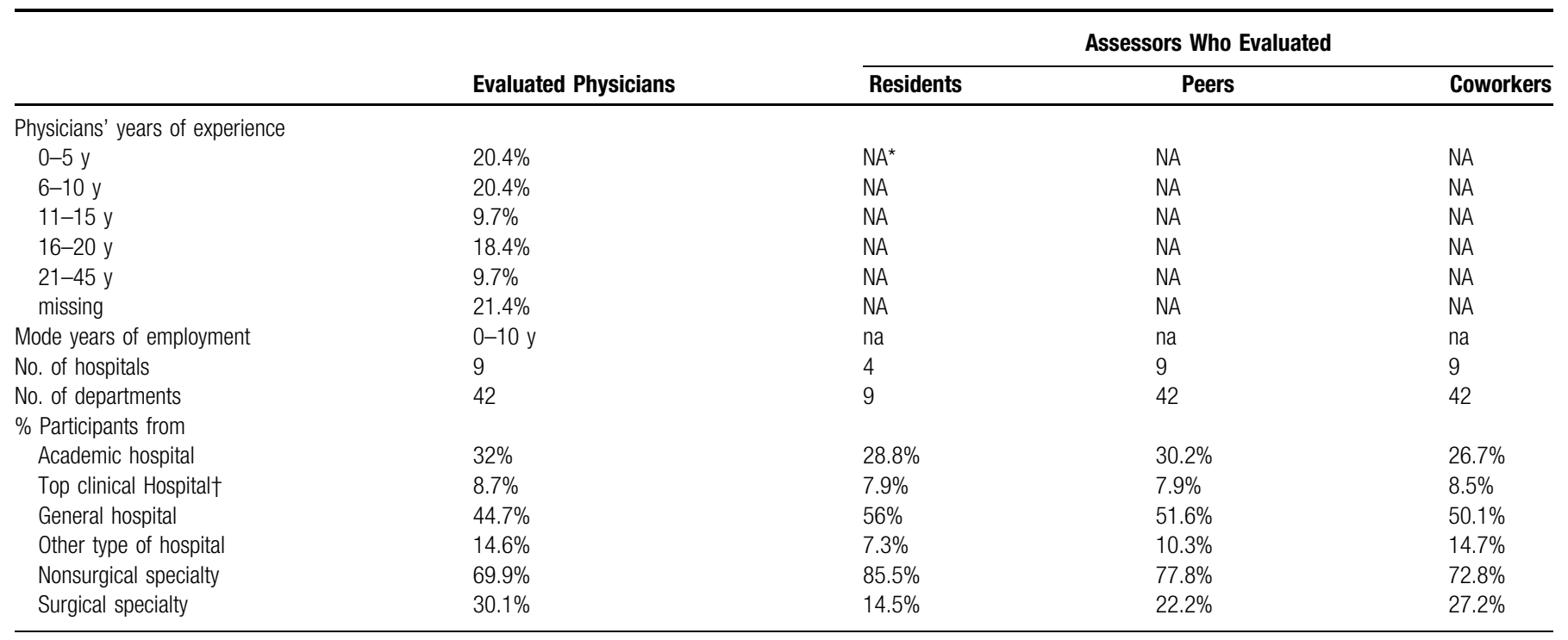

*NA indicates not available.

†Top clinical hospitals provide basic as well as complex health care procedures yet is not an academic medical center

\section{TABLE 2}

Descriptive Statistics of the Multisource Feedback Scores Given by 242 Residents, 684 Peers, and 999 Coworkers to 103 Physician During 2012-2018 for 2 MSF Evaluations

\begin{tabular}{|c|c|c|c|}
\hline & \multicolumn{3}{|c|}{ Scores Given by } \\
\hline & Residents & Peers & Coworkers \\
\hline \multicolumn{4}{|c|}{ Average score given to all physicians (SD): } \\
\hline At time 1 & $4.35(0.24)$ & $4.40(0.27)$ & $4.41(0.26)$ \\
\hline At time 2 & $4.34(0.28)$ & $4.36(0.28)$ & $4.41(0.24)$ \\
\hline \multicolumn{4}{|l|}{ PA } \\
\hline At time 1 & $4.34(0.31)$ & $4.40(0.32)$ & $4.38(0.32)$ \\
\hline At time 1 & $4.32(0.26)$ & $4.32(0.30)$ & $4.28(0.33)$ \\
\hline At time 2 & $4.30(0.24)$ & $4.29(0.32)$ & $4.32(0.31)$ \\
\hline \multicolumn{4}{|l|}{ Patient centeredness } \\
\hline At time 1 & $4.43(0.32)$ & $4.47(0.26)$ & $4.55(0.22)$ \\
\hline At time 2 & $4.42(0.33)$ & $4.45(0.28)$ & $4.53(0.23)$ \\
\hline \multicolumn{4}{|c|}{ Average score change $(\mathrm{SD})$ and $\%$ of improvement } \\
\hline Score change & $-0.01(0.25)$ & $-0.05(0.25)$ & $-0.03(0.24$ \\
\hline$\%$ of physicians improved & $50 \%$ & $44 \%$ & $48 \%$ \\
\hline \multicolumn{4}{|l|}{ Organization and (self)management } \\
\hline Score change & $-0.03(0.24)$ & $-0.02(0.28)$ & $0.03(0.30)$ \\
\hline$\%$ of physicians improved & $47 \%$ & $50 \%$ & $58 \%$ \\
\hline \multicolumn{4}{|l|}{ Patient centeredness } \\
\hline Score change & $0.00(0.30)$ & $-0.02(0.28)$ & $-0.01(0.25$ \\
\hline$\%$ of physicians improved & $52 \%$ & $47 \%$ & $47 \%$ \\
\hline
\end{tabular}

*A positive value indicates a positive score change: hence improvement in time 2 scores compared with time 1 scores. A negative value thus indicates a negative score change: a decrease in time 2 scores compared with time 1 scores. 
Significant Associations Between Self-Other Negative Discrepancies and Score Changes

Intraclass correlations for the outcome variable "score change" in the three performance domains PA, OSM, and PC were 0.21, 0.14 , and 0.20 , respectively. Hence, approximately 14 to $21 \%$ of the variability in score changes within the 3 domains was associated with differences between physicians. We, therefore, proceeded the analyses with mixed-effects models. The varyingintercept models revealed that for two performance domains (OSM and PC), negative discrepancies had a significant negative association with assessors' score changes, and the varying-slope model revealed a significant random slope for the PA domain. Testing the random intercept with random slope models to verify whether significant variation between physicians' slopes of negative discrepancies exists yielded no better fit for the OSM and PC performance domains (OSM: $\Delta \chi^{2}$ OSM $=7.79 ; \Delta \mathrm{df}=4 ; P=0.10$ and PC: $\left.\Delta \chi^{2}{ }_{\mathrm{PC}}=3.85 ; \Delta \mathrm{df}=4 ; P=0.43\right)$. The number of negative discrepancies was negatively associated with score change between time 1 and time 2 for OSM and PC (OSM: $\mathrm{b}_{\mathrm{OSM}}=-0.02$; $95 \% \mathrm{CI},-0.03$ to $-0.02 ; \mathrm{SE}=0.004 ; \mathrm{PC}: \mathrm{b}_{\mathrm{PC}}=-0.03 ; 95 \% \mathrm{CI}$, -0.03 to $-0.02 ; \mathrm{SE}=0.004)$. No significant main or interaction effect was found for the type of assessor for any of the three performance domains. The final model of the OSM and PC domains concludes that when physicians were not confronted with negative discrepancies, they showed an improvement in time 2 scores. In contrast, physicians who were confronted with 18 negative discrepancies showed a negative score change, that is, a decrease in assessors' scores at time 2; hence, they did not improve their score. For an example of these results, refer Supplemental Digital Content 1 (see Figure 2, http://links.lww.com/JCEHP/A114). Due to the nonsignificant random slopes of OSM and PC, no cross-level interaction effects were tested with physicians' years of experience for those performance domains. For PA score changes, a better fit of the random slopes model was observed $\left(\Delta \chi^{2} \mathrm{PA}=25.31, \Delta \mathrm{df}=4\right.$, $P<0.001)$ and testing the cross-level interaction of physicians' years of experience with negative discrepancies yielded significant associations. Hence, the years of experience explained the negative slopes of physicians: physicians with 6 to 10 and 16 to 20 years of experience have a negative association of negative discrepancies with score changes $\left(b_{6-10}\right.$ years' experience $=-0.03,95 \%$ CI $[-0.05$ to -0.003$] ; \mathrm{SE}=0.01 ; \mathrm{b}_{16-20}$ years' experience $=-0.03,95 \% \mathrm{CI}$ $[-0.06$ to -0.004$], \mathrm{SE}=0.01)$. Hence, experienced physicians who were confronted with more negative discrepancies improved less or even failed to improve according to all assessor groups compared with less-experienced physicians. Supplemental Digital Content 1 (see Figure 3, http://links.lww.com/JCEHP/A114) shows a visual representation of these results.

\section{Educational Importance of Findings}

The associations of negative discrepancies with score changes were substantial, as the standardized regressions showed beta's of $-0.15,-0.11$, and -0.11 for PA, OSM and PC, respectively. The final models explained $48 \%, 40 \%$, and $41 \%$ of the variance found in the differences in scores for PA, OSM, and PC, respectively, whereas negative discrepancies explained $19 \%, 13 \%$, and $14 \%$ of the variance after adjustment of covariates. The final models showed a significantly better fit than the intercept-only models (PA: $\Delta \chi^{2}$ PA $=122.38 ; \Delta d f=16 ; P<.001 ;$ OSM: $\Delta \chi^{2}$ OSM $=103.79$; $\Delta \mathrm{df}=6 ; P<.001$ and PC: $\Delta \chi^{2}$ PC $\left.=116.91 ; \Delta \mathrm{df}=6 ; P<.001\right)$. Tables 3-5 display the unstandardized regression coefficients, standardized regression coefficients, random slope variance, and random intercepts variances of the final models.

\section{DISCUSSION}

Given the importance of MSF for the evaluation and improvement of physicians' performance, this study was set up to scrutinize a key component in MSF: negative discrepancies in self-assessment and assessors' scores and their association with physicians' subsequent score changes. Because there was little insight in how these discrepancies would influence physicians' subsequent performance, we examined if physicians who were confronted with negative discrepancies would receive more positive or negative MSF scores at their second MSF evaluation. In this study, $49 \%$ of the physicians improved their total MSF score between time 1 and time 2 . This result is similar to data

TABLE 3.

Unstandardized Regression Coefficients, Standardized Regression Coefficients, and Random Intercepts Variances of the Associations Between Negative Discrepancies With Score Changes in the "Organization and (self)management" Domain

\begin{tabular}{|c|c|c|c|}
\hline & \multicolumn{3}{|c|}{ Score Changes in Organization and (self)management } \\
\hline & $\begin{array}{l}\text { Unstandardized Regression } \\
\text { Coefficients (SE) }\end{array}$ & $\begin{array}{l}\text { 95\% CI of Unstandardized Regression } \\
\text { Coefficients }\end{array}$ & $\begin{array}{c}\text { Standardized Regression } \\
\text { Coefficients }\end{array}$ \\
\hline Intercept & $.17(.04)^{\star}$ & .10 to .25 & -.001 \\
\hline Random effect of intercept (SD) & $.02(.14)$ & NA & NA \\
\hline Negative discrepancy score (0-18) & $-.02(.004)$ & -.03 to -.02 & -.11 \\
\hline Physicians' scores at time $1(1-5) \dagger$ & $-.55(.05)$ & -.65 to -.44 & -.17 \\
\hline \multicolumn{4}{|l|}{ Covariates } \\
\hline Percentage missingness on items time 1 & $-.01(.01)$ & -.04 to .01 & -.02 \\
\hline Percentage missingness on items time 2 & $.007(.02)$ & -.04 to .05 & .003 \\
\hline $\begin{array}{l}\text { Months between time } 1 \text { and time } 2 \\
\text { evaluation }\end{array}$ & $.004(.004)$ & 0.001 to .01 & .04 \\
\hline $\begin{array}{l}\text { Physicians' gender }(0=\text { male, } 1= \\
\text { female) }\end{array}$ & $-.05(.04)$ & -.13 to .03 & -.02 \\
\hline
\end{tabular}

*Bold text indicate significant values at $P<.001$.

†All variables (except the negative discrepancy score, physicians' years of experience, and physicians' gender) have been centered to the grand mean to avoid multicollineairity and to help interpret the coefficients by giving the variables a meaningful zero.

NA indicates not applicable. 
Unstandardized Regression Coefficients, Standardized Regression Coefficients, and Random Intercepts Variances of the Associations Between Negative Discrepancies With Score Changes in the "Patient-Centeredness" Domain

\begin{tabular}{|c|c|c|c|}
\hline & \multicolumn{3}{|c|}{ Score Changes in Patient Centeredness } \\
\hline & $\begin{array}{l}\text { Unstandardized Regression } \\
\text { Coefficients (SE) }\end{array}$ & $\begin{array}{c}\text { 95\% CI of Unstandardized Regression } \\
\text { Coefficients }\end{array}$ & $\begin{array}{c}\text { Standardized Regression } \\
\text { Coefficients }\end{array}$ \\
\hline Random effect of intercept (SD) & $.01(.11)$ & NA & NA \\
\hline Negative discrepancy score $(0-18)$ & $-.03(.003)$ & -.03 to -.02 & -.11 \\
\hline Physicians' scores at time $1(1-5) \dagger$ & $-.59(.05)$ & -.71 to -.49 & -.15 \\
\hline \multicolumn{4}{|l|}{ Covariates } \\
\hline $\begin{array}{l}\text { Months between time } 1 \text { and time } 2 \\
\text { evaluation }\end{array}$ & $.003(.002)$ & -0.001 to .006 & .03 \\
\hline $\begin{array}{l}\text { Physicians' gender ( } 0=\text { male, } 1= \\
\text { female) }\end{array}$ & $-.003(.03)$ & -.07 to .07 & .002 \\
\hline
\end{tabular}

*Bold text indicate significant values at $P<.001$

†All variables (except the negative discrepancy score, physicians' years of experience, and physicians' gender) have been centered to the grand mean to avoid multicollineairity and to help interpret the coefficients by giving the variables a meaningful zero.

NA indicates not applicable.

from other research on physicians' self-reported performance improvement after receiving MSF. ${ }^{15-17,19,41}$ Whether physicians improved their subsequent scores seems to be influenced by the number of negative discrepancies that physicians were con- fronted with when receiving their feedback report. The extent of performance improvement declined when physicians are confronted with more negative discrepancies, as showed by the significant negative associations. Previous research has suggested

TABLE 5.

Unstandardized Regression Coefficients, Standardized Regression Coefficients, and Random Intercepts Variances of the Associations Between Negative Discrepancies With Score Changes in the "Professional Attitude" Domain

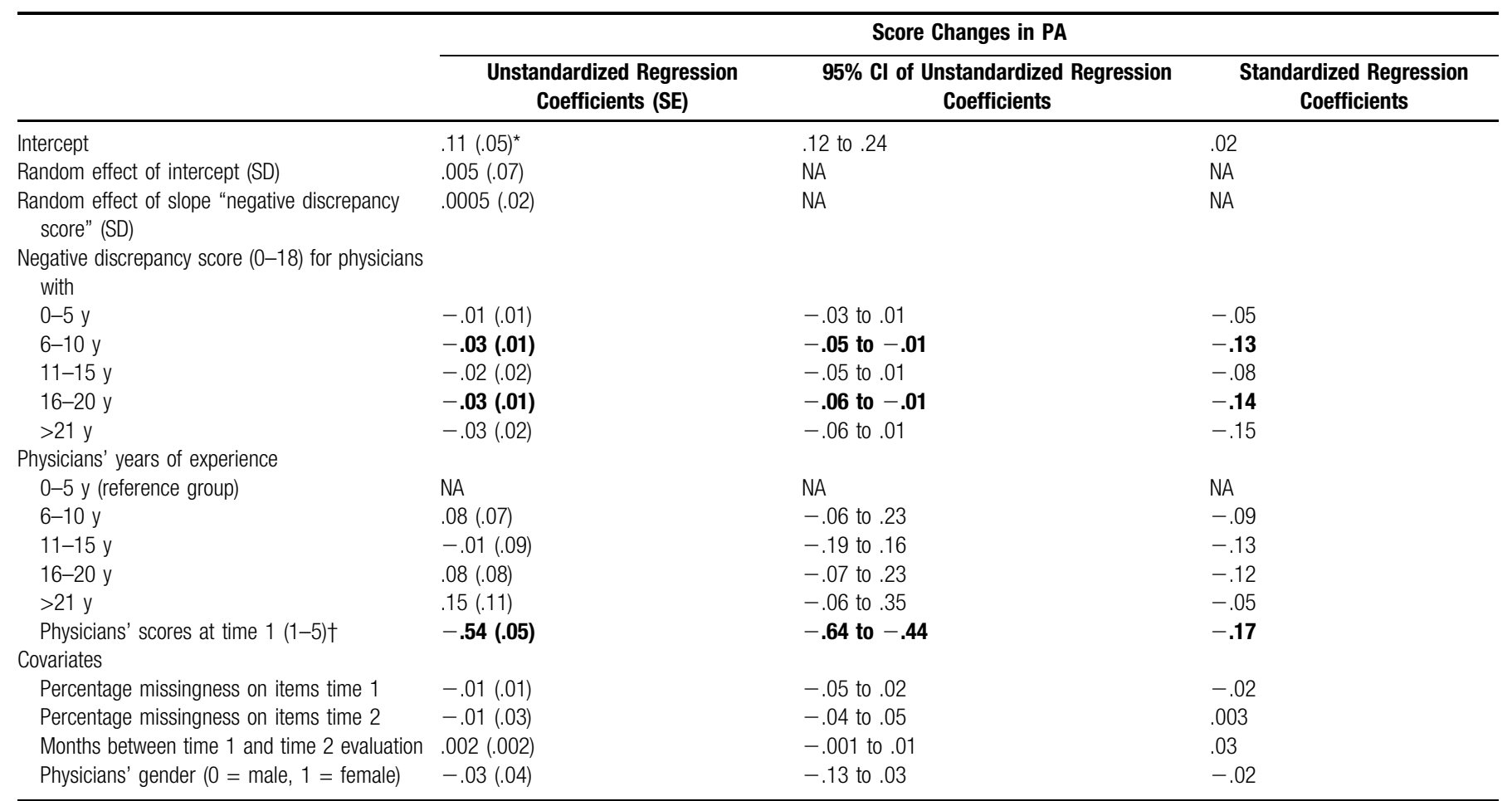

*Bold text indicate significant values at $P<.03$.

†All variables (except the negative discrepancy score, physicians' years of experience, and physicians' gender) have been centered to the grand mean to avoid multicollineairity and to help interpret the coefficients by giving the variables a meaningful zero.

MSF indicates multisource feedback; NA, not applicable. 
that the extent to which feedback is deemed valuable depends on the degree to which feedback can be reconciled with one's selfassessment. $^{21,26}$ Our research findings may point to the limit of reconcilement of feedback with self-assessment because performance seems to decline when more than half of the feedback items show negative discrepancies. Although the unstandardized regressions coefficients showed small associations, it must be kept in mind that the range of "score changes" is fairly small, and the relative importance of negative discrepancies upon "score changes" was substantial. Possible explanations of why negative discrepancies diminish the extent of performance improvement range from the feedback recipients' emotional reactions to cognitive factors. ${ }^{26}$ Physicians who overrate their performance are uninformed of their current competency and might be unaware of the standards of high performance. These physicians are perhaps less likely to achieve better performance because they simply do not know how and are reluctant to ask for additional feedback due to fear of not appearing knowledgeable. ${ }^{29}$ Furthermore, physicians' emotional responses after being confronted with negative discrepancies might also take up essential cognitive resources to adequately deal with this type of feedback. ${ }^{33}$ Especially, feedback that focuses on personal performance, such as MSF, has the potential to trigger overwhelming emotions. Uptake and use of feedback are challenging if it threatens the recipients' self-esteem or self-image, and if it elicits strong, negative emotions. ${ }^{28}$ However, our results hint at the fact that physicians' confidence might be the underlying factor in not accepting incongruent feedback. Physicians with an average total prescore of 4.2, who were confronted with more than nine negative discrepancies, showed an average performance score decline of 0.11 in the next MSF evaluation. These physicians rated themselves quite high, as an average total score of 4.2 would mean that these physicians gave themselves a score of five on multiple items, implying that they are fairly confident of their performance. Being overly confident has been found to distort acceptance of feedback, perceiving feedback as less credible and thus cause denial of feedback. ${ }^{26}$ Comparable results of the negative effects of self-overrating on subsequent MSF scores have been found in MSF research conducted in personnel psychology and medical education. Personnel managers and clinical teachers who had severely overrated their performance showed less or eventually no improvement in the subsequent scores given by assessors. $^{20,42-45}$ Further support for this reluctance to change due to physicians' confidence stems from the finding that score changes in the PA domain were not influenced by the number of negative discrepancies for physicians with less than six years of experience. Only physicians with 6 to 10 and 16 to 20 years of experience showed a significant negative association with score changes at time 2 . Because confidence is directly linked to experience $^{26}$ (the more experience, the more confident), this might indicate that too much confidence results in disregarding incongruent feedback. To find out more about the influence of physicians' confidence level and acceptance of incongruent feedback, an interesting take would be to focus on the magnitude of discrepancies as well, rather than solely the number of discrepancies. With the magnitude of discrepancies, it could be argued that the higher the magnitude, the more overconfident the physician would be. Other measures of physicians' confidence level and other assessment scores of physicians should be taken into account as well to gauge whether confident physicians are reluctant to change or whether they are unable to change. Yet to investigate this matter, future research is needed that takes a mixed-methods approach. Previous research indicated that the type of feedback provider influences the perceived credibility of feedback and thus the acceptance and use of feedback. ${ }^{27,30,31}$ In this study, we did not find significant differences among the different assessor groups in physicians' subsequent scores, neither between the associations of negative discrepancies with score changes. The more often physicians overrated their performance, the less improvement in scores was observed, according to every assessor group in every performance domain. This could mean that for these physicians, none of the assessor groups were credible enough to accept and use the feedback for performance improvement. However, the average number of negative discrepancies (6) was similar in all three assessor groups. Hence, this lack of variation could also explain why no significant assessor differences were found between the associations of negative discrepancies with score changes. These results need to be clarified in further research to investigate whether accepting negative discrepancies depends on other contextual factors. The results unfolded in this study ask for future research directed at a deeper understanding of why too many negative discrepancies cause performance decline and how to address others' feedback more effectively. This future research should include qualitative approaches to understand why specific responses are made when physicians are confronted with negative discrepancies, for example, by conducting semistructured interviews with evaluated physicians. Moreover, the content and specificity of the narrative comments that are part of MSF should be investigated and could reveal more insight into the type of feedback, the feedback provider, and the feedback recipient. ${ }^{46}$ Difficult questions regarding the effectiveness of MSF require both quantitative and qualitative research methods.

\section{LIMITATIONS}

There are some limitations to the present study. A relatively small sample of 103 physicians was included in this study. These physicians were evaluated before the mandating of participation in MSF, from 2012 to 2018. Participation in MSF assessment has only recently become mandatory for Dutch medical specialists (January 2020), which might have been the reason for the relatively small sample. Besides this relatively small sample size, we assumed that changes in scores given by residents, peers, and coworkers indicated physicians' performance change. Although the validity of the measurements is supported with evidence from the literature and empirical analyses, ${ }^{34}$ we did not ask assessors whether they noticed changes in the physicians' performance, nor did we compare their scores to other performance measurements. Combining several measurements, such as perceived performance improvement and external evaluation data, yields more insight into performance. ${ }^{47,48}$ Also, we cannot state with certainty that the MSF process or the negative self-other discrepancies caused the performance changes, as we could not include a control group in this study. This observational retrospective study merely investigated associations without a controlled postperiod. Furthermore, research has 
demonstrated that MSF does not self-evidently result in performance change, but the facilitative interview following MSF does. ${ }^{49}$ Unfortunately, the period after MSF collection has not been monitored, and hence, it was not taken into consideration how physicians discussed their feedback afterwards. Finally, the inherent limits of using a short Likert-type scale for the evaluation of practicing physicians should be mentioned. Consistent with other MSF research, most assessors gave high scores resulting in highly skewed favorable impressions of physician performance. ${ }^{50,51}$ These high scores imply that a large part of the physicians scored well above 4.0, and for them, the 5-point scale simply allows very little positive change. Indeed, we found a significant negative association between time 1 scores and subsequent score changes. Nevertheless, these physicians still have high scores at time 2 . The difficulty of detecting score change when the performance distributions are skewed is an issue known to MSF, ${ }^{47}$ and this issue was present in this study as well.

\section{IMPLICATIONS}

The results of this study imply that when physicians receive MSF, confrontation with too many negative discrepancies might be detrimental for subsequent performance scores. Performance decline seemed to be present when more than half of the feedback report's scores showed negative discrepancies. Thus, for physicians with lower assessor scores than expected from self-assessment, achieving performance improvement will be more challenging, and newer (follow-up) approaches need to be considered or even designed. Depending on the explanations of the lack of improvement in self-overrating physicians, different approaches may be needed. ${ }^{6,42}$ It seems that the follow-up should focus upon physicians' acceptance of feedback and especially on the acceptance of feedback that shows a multitude of negative discrepancies between physicians' self and assessors' scores. Helpful interventions may include raising awareness of physicians on why these negative discrepancies have occurred, assisting physicians to focus their attention on the actual feedback instead of meticulously focusing on negative discrepancies and providing facilitated follow-up on feedback uniquely tailored to (overly) confident physicians.

\section{CONCLUSION}

MSF is a popular method in the evaluation of practicing physicians' professional performance. However, there appears to be a trade-off in MSF: at a certain point, the discrepancies in given feedback may become too much for recipients to translate into performance improvement. It is essential to conduct in-depth research into the reasoning processes and confidence of recipients to reach MSF's full potential. In the end, receiving feedback is not an emotionally neutral task, and its implications are like a double-edged sword: it may help as well as hinder the improvement of physicians' performance. The goal of using MSF for the improvement of physicians' professional performance can perhaps be reached by discussing attempts to reconcile physicians' dissonances with the feedback and provide stimulating guidance for improvement.

\section{Lessons for Practice}

- The extent of physicians' performance improvement, as rated by their assessors, is likely to decline when physicians are confronted with negative discrepancies between selfassessment scores and their assessors' scores.

-When physicians are confronted with negative discrepancies on more than half of the total feedback items, their performance scores are more likely to deteriorate in the subsequent MSF evaluation, compared with the previous evaluation.

- Physicians' confidence might explain why physicians who overrate their performance do not improve their performance, and this confidence should be taken into account during the facilitative interview after MSF.

\section{ACKNOWLEDGMENTS}

The authors thank Empirion/Medox.nl for their efforts in the design and continuous technical development of the multisource feedback instrument "INCEPT" web-based application.

\section{REFERENCES}

1. Berwick DM. Era 3 for medicine and health care. JAMA. 2016;315: 1329-1330.

2. Kogan JR, Holmboe ES. Realizing the promise and importance of performance-based assessment. Teach Learn Med. 2013;25(suppl 1): S68-S74.

3. Lanier DC, Roland M, Burstin H, et al. Doctor performance and public accountability. Lancet. 2003;362:1404-1408.

4. Weiss KB. Future of board certification in a new era of public accountability. J Am Board Fam Med. 2010;23(suppl 1):S32-S39.

5. Mackillop LH, Crossley J, Vivekananda-Schmidt P, et al. A single generic multi-source feedback tool for revalidation of all UK career-grade doctors: does one size fit all? Med Teach. 2011;33:e75-83.

6. Brett JF, Atwater LE. 360 degrees feedback: accuracy, reactions, and perceptions of usefulness. J Appl Psychol. 2001;86:930-942.

7. Evans R, Elwyn G, Edwards A. Review of instruments for peer assessment of physicians. BMJ. 2004;328:1240.

8. Ramsey PG, Wenrich MD. Peer ratings. An assessment tool whose time has come. J Gen Intern Med. 1999;14:581-582.

9. Epstein RM, Hundert EM. Defining and assessing professional competence. JAMA. 2002;287:226-235.

10. Govaerts MJB, Van der Vleuten CPM, Holmboe ES. Managing tensions in assessment: moving beyond either-or thinking. Med Educ. 2019;53:6475 .

11. Whitehead CR, Hodges BD, Austin Z. Dissecting the doctor: from character to characteristics in North American medical education. Adv Health Sci Educ Theor Pract. 2013;18:687-699.

12. Davis DA, Mazmanian PE, Fordis M, et al. Accuracy of physician selfassessment compared with observed measures of competence: a systematic review. JAMA. 2006;296:1094-1102.

13. Johnson JW, Ferstl KL. The effects of interrater and self-other agreement on performance improvement following upward feedback. Pers Psychol. 1999;52:271-303.

14. Smither JW, London M, Reilly RR. Does performance improve following multisource feedback? A theoretical model, meta-analysis, and review of empirical findings. Pers Psychol. 2005;58:33-66.

15. Fidler H, Lockyer JM, Toews J, et al. Changing physicians' practices: the effect of individual feedback. Acad Med. 1999;74:702-714. 
16. Hall W, Violato C, Lewkonia R, et al. Assessment of physician performance in Alberta: the physician achievement review. Can Med Assoc J. 1999;161:52-57.

17. Lockyer J, Violato C, Fidler H. Likelihood of change: a study assessing surgeon use of multisource feedback data. Teach Learn Med. 2003;15: 168-174.

18. Overeem K, Wollersheim H, Driessen EW, et al. Doctors' perceptions of why 360-degree feedback does (not) work: a qualitative study. Med Educ. 2009;43:874-882.

19. Overeem K, Wollersheim HC, Arah OA, et al. Factors predicting doctors' reporting of performance change in response to multisource feedback. BMC Med Educ. 2012;12:52.

20. Sargeant J, Mann K, Ferrier S. Exploring family physicians' reactions to multisource feedback: perceptions of credibility and usefulness. Med Educ. 2005;39:497-504.

21. Sargeant J, Mann K, Sinclair D, et al. Understanding the influence of emotions and reflection upon multi-source feedback acceptance and use. Adv Health Sci Educ Theor Pract. 2008;13:275-288.

22. Sargeant JM, Mann KV, Van der Vleuten CPM, et al. Reflection: a link between receiving and using assessment feedback. Adv Health Sci Educ Theor Pract. 2009;14:399-410.

23. Vinod SK, Lonergan DM. Multisource feedback for radiation oncologists. J Med Imaging Radiat Oncol. 2013;57:384-389.

24. Warner DO, Sun H, Harman AE, et al. Feasibility of patient and peer surveys for Maintenance of Certification among diplomates of the American Board of Anesthesiology. J Clin Anesth. 2015;27:290-295.

25. Hattie J, Timperley H. The power of feedback. Rev Educ Res. 2007;77: 81-112.

26. Eva KW, Armson H, Holmboe E, et al. Factors influencing responsiveness to feedback: on the interplay between fear, confidence, and reasoning processes. Adv Health Sci Educ Theor Pract. 2012;17:15-26.

27. Roberts MJ, Campbell JL, Richards SH, et al. Self-other agreement in multisource feedback: the influence of doctor and rater group characteristics. J Contin Educ Health Prof. 2013;33:14-23.

28. Watling CJ, Ginsburg S. Assessment, feedback and the alchemy of learning. Med Educ. 2019;53:76-85.

29. Mann K, Van der Vleuten CPM, Eva KW, et al. Tensions in informed selfassessment: how the desire for feedback and reticence to collect and use it can conflict. Acad Med. 2011;86:1120-1127.

30. Yama BA, Hodgins M, Boydell K, et al. A qualitative exploration: questioning multisource feedback in residency education. BMC Med Educ. 2018;18:170.

31. Crossley J, Jolly B. Making sense of work-based assessment: ask the right questions, in the right way, about the right things, of the right people. Med Educ. 2012;46:28-37.

32. Brennan N, Bryce M, Pearson M, et al. Towards an understanding of how appraisal of doctors produces its effects: a realist review. Med Educ. 2017; 51:1002-1013.

33. DeNisi AS, Kluger AN. Feedback effectiveness: can 360-degree appraisals be improved?. Acad Manage Exec. 2000;14:129-139.

34. Van der Meulen MW, Boerebach BC, Smirnova A, et al. Validation of the INCEPT: a multisource feedback tool for capturing different perspectives on physicians' professional performance. J Contin Educ Health Prof. 2017;37:9-18

35. Bloch R, Norman G. Generalizability theory for the perplexed: a practical introduction and guide: AMEE Guide No. 68. Med Teach. 2012;34:960-992.

36. Boor K, Scheele F, Van der Vleuten CPM, et al. Psychometric properties of an instrument to measure the clinical learning environment. Med Educ. 2007;41:92-99.

37. Norcini JJ. Standards and reliability in evaluation: when rules of thumb don't apply. Acad Med. 1999;74:1088-1090.

38. Tabachnick BG, Fidell LS. Using Multivariate Statistics. Upper Saddle River, NJ: Pearson Education Inc.; 2013.

39. Hox JJ, Moerbeek M, Van de Schoot R. Multilevel Analysis: Techniques and Applications. New York, NY: Routledge; 2017.

40. R: A Language and Environment for Statistical Computing. [computer Program]. Version R Version 3.5.1. Vienna, Austria: R Foundation for Statistical Computing; 2018.

41. Violato C, Lockyer JM, Fidler H. Changes in performance: a 5-year longitudinal study of participants in a multi-source feedback programme. Med Educ. 2008;42:1007-1013.

42. Atwater LE, Brett JF, Charles AC. Multisource feedback: lessons learned and implications for practice. Hum Resour Manage. 2007;46:285-307.

43. Atwater LE, Waldman DA, Brett JF. Understanding and optimizing multisource feedback. Hum Resour Manage. 2002;41:193-208.

44. Boerebach BC, Arah OA, Heineman MJ, et al. The impact of resident- and self-evaluations on surgeon's subsequent teaching performance. World J Surg. 2014;38:2761-2769.

45. Ostroff C, Atwater LE, Feinberg BJ. Understanding self-other agreement: a look at rater and ratee characteristics, context, and outcomes. Pers Psychol. 2004;57:333-375.

46. van der Leeuw RM, Schipper MP, Heineman MJ, et al. Residents' narrative feedback on teaching performance of clinical teachers: analysis of the content and phrasing of suggestions for improvement. Postgrad Med J. 2016;92:145-151.

47. Boerebach BC, Arah OA, Heineman MJ, et al. Embracing the complexity of valid assessments of clinicians' performance: a call for in-depth examination of methodological and statistical contexts that affect the measurement of change. Acad Med. 2016;91:215-220.

48. Schuwirth LW, Van der Vleuten CPM. Programmatic assessment and Kane's validity perspective. Med Educ. 2012;46:38-48.

49. Sargeant J, Lockyer J, Mann K, et al. Facilitated reflective performance feedback: developing an evidence- and theory-based model that builds relationship, explores reactions and content, and coaches for performance change (R2C2). Acad Med. 2015;90:1698-1706.

50. Wright C, Richards SH, Hill JJ, et al. Multisource feedback in evaluating the performance of doctors: the example of the UK General Medical Council patient and colleague questionnaires. Acad Med. 2012;87:16681678.

51. Campbell JL, Richards SH, Dickens A, et al. Assessing the professional performance of UK doctors: an evaluation of the utility of the General Medical Council patient and colleague questionnaires. Oual Saf Health Care. 2008;17:187-193. 